\title{
BMJ Open Impact of cardiopulmonary resuscitation on a cannot intubate, cannot oxygenate condition: a randomised crossover simulation research study of the interaction between two algorithms
}

Thomas Ott (D) , Jascha Stracke, ${ }^{1}$ Susanna Sellin, ${ }^{1}$ Marc Kriege, ${ }^{1}$ Gerrit Toenges, ${ }^{2}$ Carsten Lott, ${ }^{1}$ Sebastian Kuhn, ${ }^{3}$ Kristin Engelhard ${ }^{1}$

To cite: 0 tt T, Stracke J, Sellin S, et al. Impact of cardiopulmonary resuscitation on a cannot intubate, cannot oxygenate condition: a randomised crossover simulation research study of the interaction between two algorithms. BMJ Open 2019;9:e030430. doi:10.1136/ bmjopen-2019-030430

- Prepublication history and additional material for this paper are available online. To view these files, please visit the journal online (http://dx.doi org/10.1136/bmjopen-2019030430).

Received 14 March 2019 Revised 07 October 2019 Accepted 10 October 2019

Check for updates

(c) Author(s) (or their employer(s)) 2019. Re-use permitted under CC BY-NC. No commercial re-use. See rights and permissions. Published by BMJ.

${ }^{1}$ Department of Anaesthesiology, Johannes Gutenberg Universitat Mainz, Mainz, Germany

${ }^{2}$ Institute of Medical

Biostatistics, Epidemiology and Informatics, Johannes Gutenberg Universitat Mainz, Mainz, Germany

${ }^{3}$ Department of Orthopaedics and Traumatology, Johannes Gutenberg Universitat Mainz, Mainz, Germany

Correspondence to

Dr Thomas 0tt;

ottth@uni-mainz.de

\section{ABSTRACT}

Objectives During a 'cannot intubate, cannot oxygenate' situation, asphyxia can lead to cardiac arrest. In this stressful situation, two complex algorithms facilitate decision-making to save a patient's life: difficult airway management and cardiopulmonary resuscitation. However, the extent to which competition between the two algorithms causes conflicts in the execution of pivotal treatment remains unknown. Due to the rare incidence of this situation and the very low feasibility of such an evaluation in clinical reality, we decided to perform a randomised crossover simulation research study. We propose that even experienced healthcare providers delay cricothyrotomy, a lifesaving approach, due to concurrent cardiopulmonary resuscitation in a 'cannot intubate, cannot oxygenate' situation.

Design Due to the rare incidence and dynamics of such a situation, we conducted a randomised crossover simulation research study.

Setting We collected data in our institutional simulation centre between November 2016 and November 2017. Participants We included 40 experienced staff anaesthesiologists at our tertiary university hospital centre. Intervention The participants treated two simulated patients, both requiring cricothyrotomy: one patient required cardiopulmonary resuscitation due to asphyxia, and one patient did not require cardiopulmonary resuscitation. Cardiopulmonary resuscitation was the intervention. Participants were evaluated by video records. Primary outcome measures The difference in 'time to ventilation through cricothyrotomy' between the two situations was the primary outcome measure.

Results The results of 40 participants were analysed. No carry-over effects were detected in the crossover design. During cardiopulmonary resuscitation, the median time to ventilation was $22 \mathrm{~s}$ (IQR 3-40.5) longer than that without cardiopulmonary resuscitation $(p=0.028)$, including the decision-making time.

Conclusion Cricothyrotomy, which is the most crucial treatment for cardiac arrest in a 'cannot intubate, cannot oxygenate' situation, was delayed by concurrent cardiopulmonary resuscitation. If cardiopulmonary resuscitation delays cricothyrotomy, it should be interrupted to first focus on cricothyrotomy.

\section{Strengths and limitations of this study}

- This study is the first to explore time frames of pivotal treatments that are not assessable in clinical reality and to demonstrate a crucial impact of cricothyrotomy in a 'cannot intubate, cannot oxygenate' situation on cardiopulmonary resuscitation.

- We collected data from experienced physicians that provide a high level of experience in airway management and emergency medicine.

- We chose a crossover design to minimise intraindividual differences of the participants.

- We used a simulated setting that offered realistic and standardised situational conditions.

- However, simulation research studies always lack direct translation into patient care and has to be interpreted with care.

\section{BACKGROUND}

During difficult airway management (DAM), asphyxia can lead to cardiac arrest. In this situation, the reestablishment of oxygenation has the highest priority in saving the patient's life. ${ }^{1-4}$ For standardised decision-making during a stressful 'cannot intubate, cannot oxygenate' (CICO) situation, DAM algorithms have been established suggesting cricothyrotomy as a final solution. ${ }^{2-5}$ At the same time, cardiopulmonary resuscitation (CPR) must begin which is also guided by an established algorithm. ${ }^{16}$ It is unknown whether the competition between these two pivotal algorithms leads to a delay in one of these lifesaving interventions. The survival rate after asphyxial cardiac arrest is low, and the time to re-establish sufficient oxygenation is the most relevant factor of survival. ${ }^{178}$ Therefore, this situation, which demands the application of high-level technical and non-technical skills, needs to be analysed, and the mutual influence of the two 


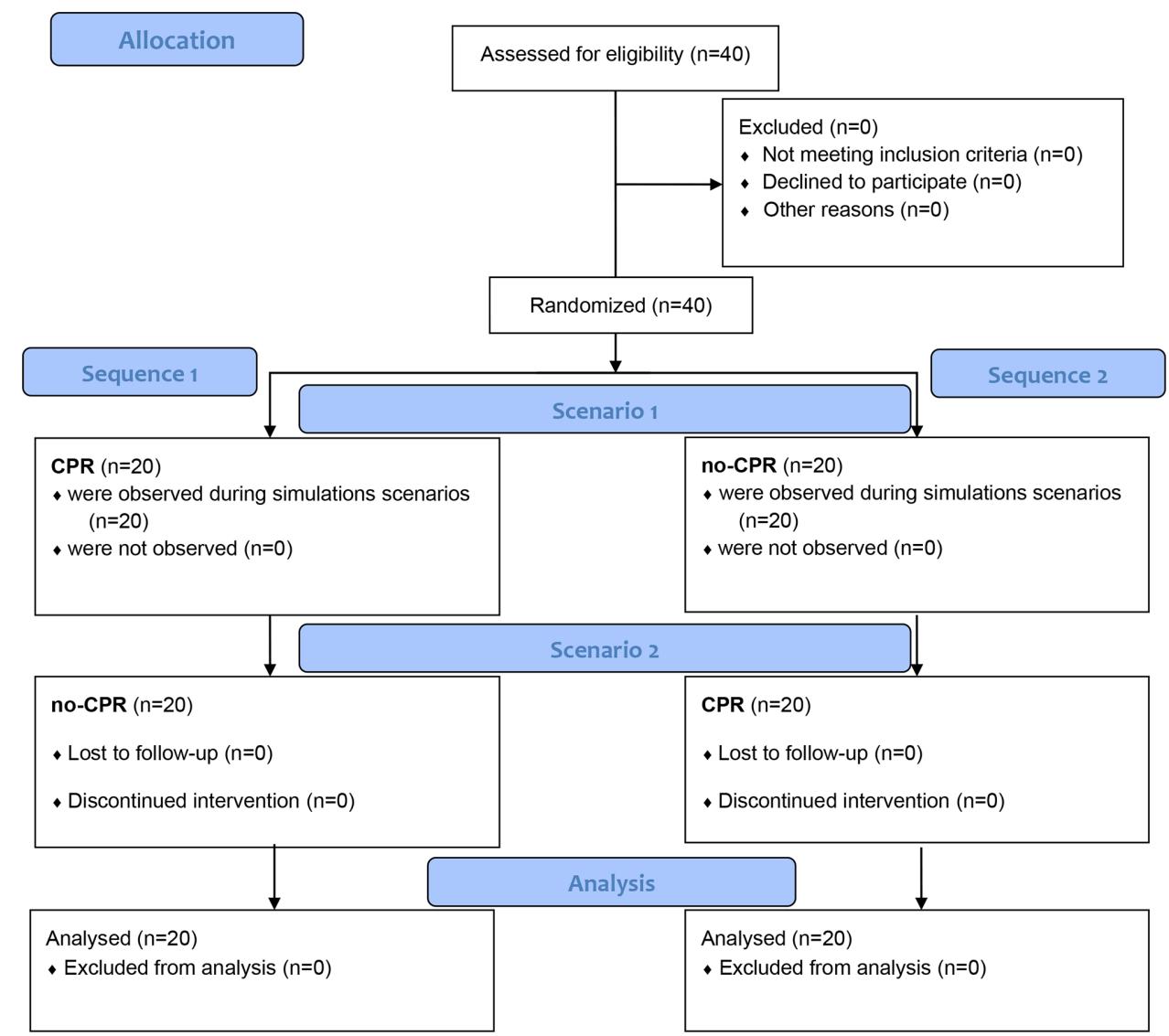

Figure 1 Consolidated Standards of Reporting Trials flow chart displaying the study design and numbers of participants allocated to the particular scenarios defining sequences (1 and 2) and periods. CPR, cardiopulmonary resuscitation.

algorithms must be characterised and possibly eliminated. ${ }^{9}$ Technical aspects, including applied airway instruments and successfully applied treatments such as cricothyrotomy, as well as non-technical aspects, including decision-making, could lead to a deeper understanding of susceptible issues in these situations. However, due to the rapid dynamics and the rare incidence of a patient requiring simultaneous cricothyrotomy and CPR, no reliable data exist regarding the time aspects of this situation; however, quicker decisionmaking and execution processes may improve neurological outcomes and survival rates in patients exhibiting asphyxia. ${ }^{51011}$ Therefore, the present simulation research study investigated these time aspects and the applied airway instruments in physicians who were well trained in DAM and CPR. ${ }^{12}$ Our hypothesis was that cricothyrotomy, the crucial treatment for survival in a CICO situation, is delayed by CPR even when these interventions are performed by experienced healthcare providers. The aim of the study was to evaluate the influence of CPR on the 'time to ventilation' through cricothyrotomy, during a CICO situation.

\section{METHODS}

\section{Ethical aspects}

After registration, the responsible ethical review committee of the Medical Association of the State Rhineland-Palatinate (Ethical Review Committee of the State Chamber of Physicians of Rhineland-Palatinate,
Deutschhausplatz 3, 55116 Mainz, Germany) confirmed that no formal ethical approval was necessary for the present study on 14 September 2016; thus, a trial registration number was not allocated by the ethical review committee. All participants signed a written informed consent form before participating in the study.

\section{Study design}

We conducted a randomised crossover study, including two situations in alternating sequences (figure 1):

1. CPR: CICO situation with asphyxial cardiac arrest.

2. No-CPR: CICO situation with severe hypoxia but without cardiac arrest.

CPR was the standardised intervention.

During the CPR situations, the CPR was explicitly performed autonomously by instructors who represented the team in the scenarios. CPR was performed according to the current European Resuscitation Council guidelines of 2015. In both scenarios, a cricothyrotomy was defined as the solution. Participants underwent both scenarios as the leading physicians. The sequence ('CPR then no-CPR' or 'no-CPR then CPR') was alternated throughout the training (figure 1, online supplementary file 1), and the frequency of the sequences resulted in a ratio of 1:1. The situations were set in two different locations according to the training content: an operating theatre and a CT suite. The period 
of the location (CT suite then operating theatre and vice versa) was reversed after each group of six participants.

\section{Institutional airway algorithms for DAM}

Our institutional algorithm for DAM was last updated in 2015 (online supplementary file 2). Every November, institutional airway training is performed, comprising workshops on all provided airway instruments and simulation scenarios for DAM. ${ }^{13}$ In every anaesthesia induction room, a poster of the algorithm is routinely installed. All but one participant had at least once attended an institutional airway training session in 2015 or earlier. One participant was employed as a staff member after the training in 2015. However, a CPR situation was simulated for the first time in this training, and the participant did not expect such a situation.

\section{Setting and study protocol}

The study was conducted in our simulation centre at a tertiary university hospital centre. Briefing of the participants before entering the simulation room included the following information for the operating theatre location: 'A team in the operating theatre calls for help due to a problem with oxygenation after the induction of general anaesthesia for an emergency inguinal herniotomy. You are the responsible anaesthesiologist in charge'. The following information was given for the CT suite location: 'A team calls for help in the CT suite because a ventilated patient was accidentally extubated during transfer onto the CT table. The patient underwent an extended tumour-related hemicolectomy and is now undergoing a sepsis-focused CT scan. You are the responsible anaesthesiologist in charge.'

A team of two instructors represented a team calling for help. One instructor representing a second-year physician was unsuccessfully trying to establish mask ventilation. The second instructor was representing a specialised anaesthesia nurse. During a CPR situation, the second instructor was performing adequate chest compressions. During a no-CPR situation, the second instructor was waiting for orders. During the CPR situations, saturation was not evident. A no-CPR situation started with an oxygen saturation of $70 \%$ that decreased by $5 \%$ every minute, whereas the other vital signs were modified according to a standardised protocol. Standard airway and anaesthesia trolleys were provided. All instruments and assistance required by the participants were provided immediately. The instructors were not allowed to help regarding any medical decisions. The study protocol is available in online supplementary file 3 .

CPR was performed according to a pulseless electrical activity algorithm by the instructors, who were certified advanced life support providers. After cricothyrotomy was performed and ventilation was established, restoration of spontaneous circulation occurred within 1 min during a CPR situation, whereas vital signs improved within 1 min in a no-CPR situation.

\section{Simulator}

The adult high-fidelity simulator SimMan (Laerdal medical AS, Stavanger, Norway) offers sufficient conditions concerning the airway anatomy. ${ }^{14}$ The airway of the simulator was modified by the producer's default settings, as follows: pharyngeal obstruction, tongue swelling, reduced range of head reclining and jaw trismus.

\section{Participants}

Between November 2016 and November 2017, we enrolled a total of 40 staff anaesthesiologists at our tertiary university hospital centre. The eligibility criterion was at least 3 years of experience. The physicians became familiarised with the simulator and the environment during a 15 min orientation session. Randomisation concerning the sequence (CPR then no-CPR or no-CRP then CPR) was performed by one author (TO); tickets with names of the participants were randomly drawn out of a black box to allocate the participants into morning and afternoon blocks. The sequence was defined by the particular block in a predefined table (online supplementary file 1). Blinding of the participants was ensured by withholding all information about the study aim until the entire data collection was completed. Participants participated in the simulation sessions during core working hours. Thus, participants were committed to secrecy concerning the content of the scenarios by a codex and did not discuss any of the scenarios.

\section{Data collection}

A two-perspective video-recording was started after briefing prior to each scenario. Perspective one was a view of the head of the manikin, and perspective two was a general view of the scenario. One author (JS) evaluated all videorecordings after receiving supervision during the first 12 videos by another author (TO) who is an anaesthesiologist consultant. A stopwatch was started as soon as the participant entered perspective one. The following items were documented: (1) 'Time to decision' in seconds was defined by the time at which the decision to perform a cricothyrotomy was articulated by the participant. (2) 'Time to start' was defined by the time at which the first instrument for cricothyrotomy touched the skin of the simulator. (3) 'Time to ventilation' was defined by the time at which the chest first rose due to ventilation after cricothyrotomy. ${ }^{15}$ The 'time of performance' of the cricothyrotomy was calculated by subtracting the 'time to start' from the 'time to ventilation'. The first and last airway instrument applied before cricothyrotomy and the method of cricothyrotomy (surgical or puncture-based) were recorded.

\section{Statistics}

To reduce the influence of confounding variables, we used a crossover design for the assessment of the time difference between the CPR ('intervention') and the no-CPR ('baseline') situation. For sample size planning, we initially expected a value of $20 \mathrm{~s}$, for both the mean and the SD of the time difference between CPR and no-CPR. That is, the study was powered for an effect size of 1 . These values were 
assumed based on clinical experience and not derived from pilot data. The study was conducted with 20 participants in each sequence, as this sample size resulted in a power of over $85 \%$ if the assumptions above held true. Each particular time-to-event endpoint was analysed in the same manner. To evaluate the within-subject time difference, differences between the time needed in period 1 and the time needed in period 2 were compared among the two sequence groups ('CPR then no-CPR' and 'no-CPR then CPR') using a MannWhitney $\mathrm{U}$ test. The median time difference between the CPR and no-CPR situations was evaluated with the HodgesLehmann estimate and its related CI. All these analyses are only valid if no carry-over effects are present. To identify such a confounding effect, a Mann-Whitney U test comparing the total within-subject sum of times among the two sequence groups was performed as a pretest. This type of analysis might be at first counterintuitive; however, simplifying the analysis by applying standard methodology for the comparison of two dependent samples (for example, a Wilcoxonsign-rank test or a t-test for the comparison of the CPR vs no-CPR situations) may cause misleading results.

The primary outcome (confirmatory analysis) was the 'time to ventilation' after cricothyrotomy, which was compared between the CPR and no-CPR situations. The secondary outcomes (descriptive analysis) during the scenario were 'time to decision', 'time to start', 'time of performance' of cricothyrotomy, and the first and last applied instruments (table 1). According to the sequence group and period number, the time-to-event outcomes of each endpoint were classified into four groups. Data are described by the median, minimum (min), first quartile (Q1), third quartile (Q3) and maximum (max) or percentage of the particular sample. As an additional secondary outcome, we compared the distribution of the cricothyrotomy method (surgical vs puncture-based), a binary variable, between the two exposures (CPR vs no-CPR) by using a McNemar test.

All reported $\mathrm{p}$ values resulted from two-sided tests. For the primary outcome, $\mathrm{p} \leq 0.05$ in the CPR vs no-CPR comparison was considered to be significant. The pretest (for the primary outcome) and all tests related to the secondary outcomes had an exploratory purpose. Thus, their $\mathrm{p}$ values are given for descriptive reasons only and have to be interpreted carefully.

We used R V.3.3.1 (R Foundation for Statistical Computing, Vienna, Austria), IBM SPSS Statistics V.23 and Microsoft Excel 2016 (Microsoft, Redmond, USA) for data management and analysis.

\section{Patient and public involvement}

Due to the topic of the study and the dynamics and incidence of the clinical events evaluated, we did not involve the public or patients in the present study.

\section{RESULTS}

\section{Demographics}

The median amount of anaesthesia experience among the participants was 7.5 years (IQR 6-11). In addition,
Table 1 Demographics of the participants

\begin{tabular}{|c|c|c|}
\hline Item and category & $\begin{array}{l}\mathrm{N} \\
\text { (participants) }\end{array}$ & $\begin{array}{l}\text { Percentage of } \\
\text { the participants }\end{array}$ \\
\hline \multicolumn{3}{|c|}{ Years of anaesthesiology experience } \\
\hline $3-4$ & 2 & 5 \\
\hline $5-6$ & 12 & 30 \\
\hline $7-8$ & 9 & 22.5 \\
\hline $9-10$ & 6 & 15 \\
\hline $11-13$ & 5 & 12.5 \\
\hline $13-21$ & 6 & 15 \\
\hline \multicolumn{3}{|l|}{ Sex } \\
\hline Female & 17 & 42.5 \\
\hline Male & 23 & 57.5 \\
\hline \multicolumn{3}{|c|}{ Number of cricothyrotomies assisted by the participant } \\
\hline 0 & 24 & 60 \\
\hline$<3$ & 9 & 22.5 \\
\hline $3-6$ & 4 & 10 \\
\hline$>6$ & 2 & 5 \\
\hline Data missing & 1 & 2.5 \\
\hline \multicolumn{3}{|c|}{ Number cricothyrotomies performed by the participant } \\
\hline 0 & 28 & 70 \\
\hline$<3$ & 9 & 22.5 \\
\hline $3-6$ & 1 & 2.5 \\
\hline$>6$ & 1 & 2.5 \\
\hline Data missing & 1 & 2.5 \\
\hline
\end{tabular}

all participants were prehospital emergency medicine providers. Further details are displayed in table 1.

\section{Primary endpoint}

Ventilation through cricothyrotomy was achieved $22 \mathrm{~s}$ earlier during the no-CPR situation than during the CPR situation ( $\mathrm{p}=0.028$ ) (table 2, figure 2 , online supplementary file 4$)$.

\section{Secondary endpoints}

The overall median time to decision was $136 \mathrm{~s}$, the median time to start was $158 \mathrm{~s}$, the median time to ventilation was $210 \mathrm{~s}$ and the median performance time of cricothyrotomy was $43 \mathrm{~s}$. The absolute 'time to decision' was $9 \mathrm{~s}$ shorter $(\mathrm{p}=0.273)$, the absolute 'time to start cricothyrotomy' was $14 \mathrm{~s}$ shorter $(\mathrm{p}=0.076)$, and the absolute 'time of performance' was $4 \mathrm{~s}$ shorter $(\mathrm{p}=0.213$ ) (table 2 , online supplementary file 4) during the no-CPR situation than during the CPR situation. However, there were no statistical differences between the CPR and no-CPR situations concerning these times.

The pretests of the time-to-event outcomes did not indicate that our results were confounded by carry-overeffects (online supplementary file 4).

The first and last instruments used during the scenarios are listed in table 3 . 
Table 2 Data from the crossover design analysis

\begin{tabular}{|c|c|c|}
\hline Category & Sequence 1 & Sequence 2 \\
\hline \multicolumn{3}{|c|}{ Time to ventilation (primary outcome) } \\
\hline Period 1 & 217 & 173.5 \\
\hline Period 2 & 196.5 & 217.5 \\
\hline HL estimate & \multicolumn{2}{|r|}{22} \\
\hline$P$ value & \multicolumn{2}{|r|}{0.028} \\
\hline \multicolumn{3}{|c|}{ Time to decision (secondary outcome) } \\
\hline Period 1 & 134.5 & 120 \\
\hline Period 2 & 130.5 & 159 \\
\hline HL estimate & \multicolumn{2}{|r|}{9} \\
\hline$P$ value & \multicolumn{2}{|r|}{0.273} \\
\hline \multicolumn{3}{|c|}{ Time to start cricothyrotomy (secondary outcome) } \\
\hline Period 1 & 162.5 & 141.5 \\
\hline Period 2 & 150.5 & 183.5 \\
\hline HL estimate & \multicolumn{2}{|r|}{14} \\
\hline$P$ value & \multicolumn{2}{|r|}{0.076} \\
\hline \multicolumn{3}{|c|}{$\begin{array}{l}\text { Time of performance (time to ventilation-time to start) } \\
\text { (secondary outcome) }\end{array}$} \\
\hline Period 1 & 43 & 35.5 \\
\hline Period 2 & 61.5 & 33.5 \\
\hline HL estimate & \multicolumn{2}{|r|}{4} \\
\hline $\mathrm{P}$ value & \multicolumn{2}{|r|}{0.213} \\
\hline
\end{tabular}

Further details of the results are displayed in the extended version of table 2 in online supplementary file 4.

The HL estimate for the median time difference (in seconds) between CPR and no-CRP with the $95 \% \mathrm{Cl}$ is provided with the corresponding $p$ value of the Mann-Whitney $U$ test.

The times are displayed as medians in seconds.

Periods 1 and 2 define the periods of the scenarios for each participant: CPR then no-CPR and vice versa.

Sequence 1 is as follows: period 1, CPR; period 2, no-CPR. Sequence 2 is as follows: period 1, no-CPR; period 2, CPR. CRP, cardiopulmonary resuscitation; HL, Hodges-Lehmann.
Table 3 First instrument and last instrument applied before cricothyrotomy in the CPR situation and in the no-CPR situation percentage and number ( $n$ ) of airway instruments applied in the first attempt and the last attempt before cricothyrotomy during the CPR and no-CPR situations

\section{CPR situation}

\begin{tabular}{|c|c|c|}
\hline \multirow[b]{2}{*}{ Instruments } & First attempt & Last attempt \\
\hline & $\%$ (n of 40 ) & $\%\left(n\right.$ of $\left.39^{\star}\right)$ \\
\hline Face mask & $55(22)$ & $20.5(8)$ \\
\hline Direct laryngoscope & $27.5(11)$ & $15.4(6)$ \\
\hline Videolaryngoscope & $5(2)$ & $23.1(9)$ \\
\hline Laryngeal mask & $7.5(3)$ & $15.4(6)$ \\
\hline $\begin{array}{l}\text { Flexible intubation } \\
\text { endoscope }\end{array}$ & $2.5(1)$ & $25.6(10)$ \\
\hline Cricothyrotomy & $2.5(1)$ & $0(0)$ \\
\hline \multicolumn{3}{|l|}{ No-CPR situation } \\
\hline \multirow[b]{2}{*}{ Instruments } & First attempt & Last attempt \\
\hline & $\%$ (n of 40 ) & $\%$ (n of 40$)$ \\
\hline Face mask & $62.5(25)$ & $12.5(5)$ \\
\hline Laryngeal mask & $17.5(7)$ & $2.5(1)$ \\
\hline Direct laryngoscope & $12.5(5)$ & $35(14)$ \\
\hline Videolaryngoscope & $7.5(3)$ & $37.5(15)$ \\
\hline $\begin{array}{l}\text { Flexible intubation } \\
\text { endoscope }\end{array}$ & $0(0)$ & $12.5(5)$ \\
\hline
\end{tabular}

During the CPR scenario, $65 \%$ of the participants performed a surgical cricothyrotomy, and $35 \%$ performed a puncture cricothyrotomy; during the no-CPR situation, $58 \%$ performed a surgical cricothyrotomy, and $42 \%$ performed a puncture-based cricothyrotomy $(p=0.453)$.

*Data were missing for one participant.

$\mathrm{CPR}$, cardiopulmonary resuscitation.

\section{DISCUSSION}

The present study shows that cricothyrotomy, which is the most crucial treatment in a CICO situation, was delayed by $22 \mathrm{~s}$ due to the concurrence of CPR. This

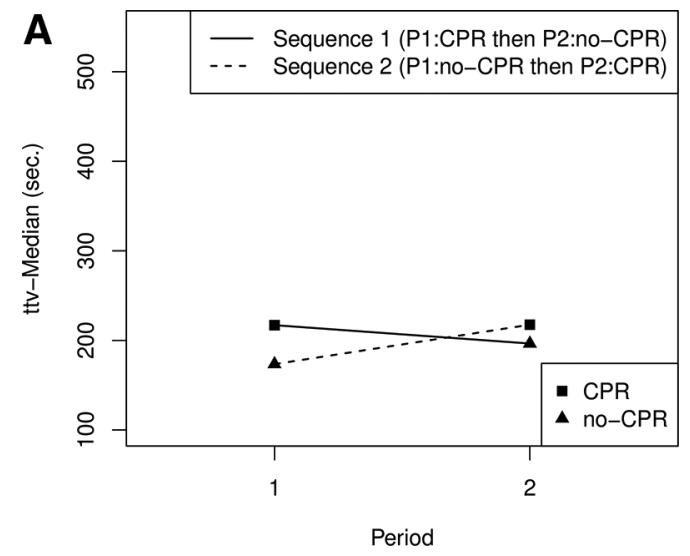

Figure 2 (A) Crossover diagram for time to ventilation (ttv). Abscissa: periods 1 and 2. Ordinate: ttv-median (s) which indicates the time to ventilation in seconds after cricothyrotomy. Quadrat: ttv during the cardiopulmonary resuscitation (CPR) situation. Triangle: ttv during the no-CPR situation. Continuous line: sequence 1. Dashed line: sequence 2. (B) Box plot of the ttv. Abscissa: periods 1 and 2. Ordinate: ttv (s) which indicates the ttv in seconds after cricothyrotomy. 
situation was evaluated in a simulated environment with physicians trained in DAM. The delay was evident, even though the participants did not have to perform CPR by themselves. Most likely, the delay in a real clinical setting is even longer than $22 \mathrm{~s}$, and the time to ventilation, including the decision-making time, probably exceeds $210 \mathrm{~s}$; in the present study, the expectations and preliminaries of the experienced airway providers who attended a simulation session about DAM most likely accelerated the application of the DAM algorithms. Additionally, in clinical practice, the decision to perform a cricothyrotomy likely takes longer due to the higher levels of inhibition concerning invasive procedures in a patient than those concerning procedures in a manikin. For the first time, the mutual influence of two pivotal lifesaving algorithms (DAM and CPR) has been characterised. The results justify the demand for stringent execution of an appropriate DAM protocol even when resuscitation is ongoing and validate courageous decision-making under imminent asphyxia.

CPR is a distractor during DAM; in the present study, the time to ventilation after a cricothyrotomy was delayed by CPR. At first glance, this result sounds trivial, particularly because the chest movements caused by CPR are likely to interfere with the evaluation of the airway conditions. However, DAM and CPR both demand a considerable amount of cognitive, communicative and organisational effort in decision-making as well as execution. Algorithms normally facilitate the execution of complex and demanding procedures which becomes difficult when two algorithms must be applied simultaneously. In the case of an asphyxial cardiac arrest in a CICO situation, we propose that chest compressions only promote the circulation of anoxic blood which is ineffective. Therefore, an interruption of chest compressions might foster the treatment of the potentially reversible causes of hypoxic cardiac arrest as soon as a CICO situation is diagnosed.

The time to ventilation was the primary endpoint in the present study, which comprised the diagnosis of a CICO condition, decision-making and performance of cricothyrotomy in less than $4 \mathrm{~min}$. The duration of each process (decision-making, starting cricothyrotomy and performing cricothyrotomy) was longer during CPR situations; however, they were not statistically different. Therefore, the significant delay of $22 \mathrm{~s}$ to perform cricothyrotomy is a summation of the decision-making, starting and performance delays. Further evaluations of decision-making within DAM are desirable to yield an understanding of potential improvements and promote the survival of individuals under asphyxia. Decisionmaking might have a higher impact on survival than cricothyrotomy techniques. ${ }^{5}$

In the present simulation research study, more than $50 \%$ of the participants performed a surgical cricothyrotomy, while the others used a Melker Set. The median time for the execution of the cricothyrotomy itself was $43 \mathrm{~s}$. This result is comparable with that of a previous simulation research study in which seventy experienced physicians needed $48 \mathrm{~s}$ for a cricothyrotomy after they were called into an ongoing scenario with an unanticipated difficult airway. ${ }^{16}$ These participants had to perform a puncture-based cricothyrotomy using a different but comparable set of instruments than that used in the present study. During a scenario with initially higher oxygen saturation but with faster desaturation, 36 experienced anaesthesiologists needed $100 \mathrm{~s}$ after the first grasp of any equipment in the available Melker Set. ${ }^{17}$ In the present study, the time started when any equipment first contacted the neck of the simulator, which might partially explain the discrepancy in the time to ventilation through cricothyrotomy between the two studies. In another study, this duration was even longer (183s), but the measurement started at the beginning of the scenario, and the physicians were from different disciplines and had various qualification levels. ${ }^{18}$ The setting of the vital signs of the simulator in that study were very similar to those in our scenario. However, the initial situation was different: one of the instructors in the scenario represented a senior anaesthetic registrar and stated a CICO situation to the participant at the start of the scenario. In contrast to the methods of the present study, the participant did not need to diagnose the situation. This difference in methods might explain the shorter time to oxygenation in that study (183s) than in the present study (210s). However, in contrast to the present investigation, neither of these studies evaluated the time for decision-making or the influence of CPR on DAM. In conclusion, the time for cricothyrotomy in the present study is comparable with that of other studies as long as similar settings and definitions were used. However, it became obvious that in clinical settings, DAM and cricothyrotomy in particular should be performed by a trained and determined physician. ${ }^{19-22}$ However, simulation remains the only method to train and evaluate such aspects of DAM.

Algorithms for DAM suggest the use of different devices for airway management but limit the number of intubation attempts at the same time..$^{2-423-25}$ The face mask is recommended as the first-choice instrument, as it has the broadest distribution to provide ventilation at any level of medical qualification..$^{2-4}$ In accordance with our institutional DAM algorithm, in the present study, the face mask was used by the vast majority of the participants as the first instrument to evaluate the airway condition. However, the settings of the simulator did not allow successful ventilation with the face mask. Therefore, the second most frequently used device by the participants was a Macintosh laryngoscope during CPR and a laryngeal mask during no-CPR situations. First published in 1943, the Macintosh laryngoscope is currently the most frequently applied and most available instrument for tracheal intubation. ${ }^{26} 27$ Experienced providers may quickly detect causes of obstructions in the upper airway using a Macintosh laryngoscope. The most frequently used instrument just before 
cricothyrotomy was a videolaryngoscope. This is an increasingly popular method for intubation offering better and faster visualisation of the laryngeal situs for the provider and the assisting staff than direct laryngoscopy does. ${ }^{27-31}$ All applied instruments are part of most DAM algorithms. ${ }^{2-4} 23-25$ Providers preferably should choose the instruments that are most quickly available, that have the highest probability of success and that they are most familiar with.

\section{Limitations}

Translation of simulation research results to patient care is difficult to validate. ${ }^{12}$ The incidence of cardiac arrest during DAM is rare and cannot be evaluated in a clinical prospective study. Thus, we decided to perform a simulation research study to evaluate the fundamental interactions in such situations in a standardised setting. Whether the findings of the present study can be generalised to real scenarios cannot be answered, but simulation is suitable to generate an idea of the effects of both algorithms. ${ }^{10} 11$ Therefore, future investigations are required to evaluate whether an interruption of CPR during cricothyrotomy should generally be recommended. Furthermore, research should focus on whether improved decision-making in a CICO situation might lead to a better survival of patients under these conditions, rather than focusing on technical aspects.

The demand for cricothyrotomy in clinical reality is rare. ${ }^{24}$ However, in the present study, 11 of 40 participants already performed a cricothyrotomy in real patient care. Data were collected by an anonymised demographic questionnaire using the categories cited in table 1. All participants worked at our tertiary university hospital centre mostly as anaesthesiological supervisors. All but one participant was active in in-hospital and out-of-hospital emergency medicine, covering approximately 68000 in-hospital patients a year. However, the number of individually protocolled incidents of cricothyrotomy was surprisingly high in our sample. This may have caused a potential bias of our sample, as experienced providers could have more quickly decided to perform a cricothyrotomy than average. Therefore, the generalisability is limited to experienced samples.

Simulation research offers the advantage of standardisation and the opportunity to evaluate rare conditions. ${ }^{32}$ Time is one of the most critical issues in airway management, and time is difficult to evaluate under emergency conditions. Thus, we launched the present study in a simulated environment to elucidate this aspect of airway management. ${ }^{21} 33$

\section{CONCLUSIONS}

In a simulator, even experienced and trained airway providers delay cricothyrotomy during concurrent CPR. This finding was evident, even though cricothyrotomy is the most crucial treatment in saving a patient's life in a CICO situation. If CPR delays the execution of cricothyrotomy and is a potentially reversible cause of cardiac arrest, it should be interrupted to prioritise cricothyrotomy.

Acknowledgements We sincerely thank the Chair of our department and the management staff, who greatly supported the organisation and coordination of the participants and instructors in our study. This manuscript contains portions of the doctoral thesis of Jascha Stracke, University Medical Centre of the Johannes Gutenberg University, Mainz, Germany. Parts of the study were presented as a scientific poster during the annual congress of the German Society of Anaesthesiology and Intensive Care Medicine (Deutsche Gesellschaft für Anästhesiologie und Intensivmedizin) on 25 April 2018, DAC (Deutscher Anästhesie Congress), Nürnberg, Germany (http://www.dac2018.de/content.php?fksart=10\& topic $=$ ePoster).

Contributors All authors have made substantial intellectual contributions to this study according to the ICMJE guidelines. The following list contains only the core areas of contribution of the individual authors. Conception and design of the study: TO, JS, SS, MK, CL. Acquisition of data: TO, JS, SS. Analysis and interpretation: GT, TO, KE, CL, SK, JS. Draft of the manuscript: TO, KE, SS, MK, CL, SK. All authors approved the final manuscript and agreed to be accountable for all aspects of the work.

Funding The authors have not declared a specific grant for this research from any funding agency in the public, commercial or not-for-profit sectors.

Competing interests None declared.

Patient consent for publication Not required.

Provenance and peer review Not commissioned; externally peer reviewed.

Data availability statement Data are available in a public, open access repository: doi:10.5061/dryad.qv9s4mw9d

Open access This is an open access article distributed in accordance with the Creative Commons Attribution Non Commercial (CC BY-NC 4.0) license, which permits others to distribute, remix, adapt, build upon this work non-commercially, and license their derivative works on different terms, provided the original work is properly cited, appropriate credit is given, any changes made indicated, and the use is non-commercial. See: http://creativecommons.org/licenses/by-nc/4.0/.

ORCID iD

Thomas Ott http://orcid.org/0000-0001-7510-7679

\section{REFERENCES}

1 Truhlár A, Deakin CD, Soar J, et al. European resuscitation Council guidelines for resuscitation 2015: section 4. cardiac arrest in special circumstances. Resuscitation 2015;95:148-201.

2 Frerk C, Mitchell VS, McNarry AF, et al. Difficult airway Society 2015 guidelines for management of unanticipated difficult intubation in adults. Br J Anaesth 2015;115:827-48.

3 Piepho T, Cavus E, Noppens R, et al. [S1 guidelines on airway management]. Anaesthesist 2015;64:859-73.

4 Apfelbaum JL, Hagberg CA, Caplan RA, et al. Practice guidelines for management of the difficult airway: an updated report by the American Society of Anesthesiologists Task force on management of the difficult airway. Anesthesiology 2013;118:251-70.

5 Stiegler MP, Ruskin KJ. Decision-Making and safety in anesthesiology. Curr Opin Anaesthesiol 2012;25:1-9.

6 Monsieurs KG, Nolan JP, Bossaert LL, et al. European resuscitation Council guidelines for resuscitation 2015: section 1. executive summary. Resuscitation 2015;95:1-80.

7 Varvarousis D, Varvarousi G, lacovidou N, et al. The pathophysiologies of asphyxial vs dysrhythmic cardiac arrest: implications for resuscitation and post-event management. Am J Emerg Med 2015;33:1297-304.

8 Graham DB, Eastman AL, Aldy KN, et al. Outcomes and long term follow-up after emergent cricothyroidotomy: is routine conversion to tracheostomy necessary? Am Surg 2011;77:1707-11.

9 Doumouras AG, Hamidi M, Lung K, et al. Non-Technical skills of surgeons and anaesthetists in simulated operating theatre crises. $\mathrm{Br}$ J Surg 2017;104:1028-36.

10 Cook TM, Woodall N, Frerk C, et al. Major complications of airway management in the UK: results of the fourth national audit project of 
the Royal College of anaesthetists and the difficult airway Society. Part 1: anaesthesia. Br J Anaesth 2011;106:617-31.

11 Cook TM, Woodall N, Harper J, et al. Major complications of airway management in the UK: results of the fourth national audit project of the Royal College of anaesthetists and the difficult airway Society. Part 2: intensive care and emergency departments. Br J Anaesth 2011;106:632-42.

12 Cheng A, Kessler D, Mackinnon R, et al. Reporting guidelines for health care simulation research: extensions to the CONSORT and STROBE statements. Adv Simul 2016;1.

13 Ott T, Truschinski K, Kriege M, et al. [Algorithm for securing an unexpected difficult airway : User analysis on a simulator]. Anaesthesist 2018;67:18-26.

14 Schalk R, Eichler K, Bergold MN, et al. A radiographic comparison of human airway anatomy and airway manikins--Implications for manikin-based testing of artificial airways. Resuscitation 2015;92:129-36.

15 Perkins GD, Handley AJ, Koster RW, et al. European resuscitation Council guidelines for resuscitation 2015: section 2. adult basic life support and automated external defibrillation. Resuscitation 2015;95:81-99.

16 John B, Suri I, Hillermann C, et al. Comparison of cricothyroidotomy on manikin vs. simulator: a randomised cross-over study. Anaesthesia 2007;62:1029-32.

17 Siu LW, Boet S, Borges BCR, et al. High-Fidelity simulation demonstrates the influence of anesthesiologists' age and years from residency on emergency cricothyroidotomy skills. Anesth Analg 2010;111:955-60.

18 Marshall SD, Mehra R. The effects of a displayed cognitive aid on non-technical skills in a simulated 'can't intubate, can't oxygenate' crisis. Anaesthesia 2014;69:669-77.

19 Lott C, Araujo R, Cassar MR, et al. The European trauma course (etc) and the team approach: past, present and future. Resuscitation 2009;80:1192-6.

20 Greif R, Lockey AS, Conaghan P, et al. European resuscitation Council guidelines for resuscitation 2015: section 10. education and implementation of resuscitation. Resuscitation 2015;95:288-301.

21 Harvey R, Foulds L, Housden T, et al. The impact of didactic readaloud action cards on the performance of cannula cricothyroidotomy in a simulated 'can't intubate can't oxygenate' scenario. Anaesthesia 2017;72:343-9.
22 Chrisman L, King W, Wimble K, et al. Surgicric 2: a comparative bench study with two established emergency cricothyroidotomy techniques in a porcine model. Br J Anaesth 2016;117:236-42.

23 Japanese Society of Anesthesiologists. JSA airway management guideline 2014: to improve the safety of induction of anesthesia. $J$ Anesth 2014;28:482-93.

24 Law JA, Broemling N, Cooper RM, et al. The difficult airway with recommendations for management - Part 1 - difficult tracheal intubation encountered in an unconscious/induced patient. Can $J$ Anesth/J Can Anesth 2013;60:1089-118.

25 Myatra SN, Shah A, Kundra P, et al. All India difficult airway association 2016 guidelines for the management of unanticipated difficult tracheal intubation in adults. Indian $J$ Anaesth 2016;60:885-98.

26 Macintosh RR. A new laryngoscope. The Lancet 1943;241.

27 Lewis SR, Butler AR, Parker J, et al. Videolaryngoscopy versus direct laryngoscopy for adult patients requiring tracheal intubation. Cochrane Database Syst Rev 2016;113

28 Goto Y, Goto T, Hagiwara Y, et al. Techniques and outcomes of emergency airway management in Japan: an analysis of two multicentre prospective observational studies, 2010-2016. Resuscitation 2017;114:14-20.

29 Kriege M, Alflen C, Tzanova I, et al. Evaluation of the McGrath MAC and Maclntosh laryngoscope for tracheal intubation in 2000 patients undergoing general anaesthesia: the randomised multicentre EMMA trial study protocol. BMJ Open 2017;7:e016907.

30 Cook TM, Kelly FE. A national survey of videolaryngoscopy in the United Kingdom. Br J Anaesth 2017;118:593-600.

31 Park SO, Kim JW, Na JH, et al. Video laryngoscopy improves the first-attempt success in endotracheal intubation during cardiopulmonary resuscitation among novice physicians. Resuscitation 2015;89:188-94.

32 Petrosoniak A, Ryzynski A, Lebovic G, et al. Cricothyroidotomy in situ simulation curriculum (CRIC study): training residents for rare procedures. Simul Healthc 2017;12:76-82.

33 Hubert V, Duwat A, Deransy R, et al. Effect of simulation training on compliance with difficult airway management algorithms, technical ability, and skills retention for emergency cricothyrotomy. Anesthesiology 2014;120:999-1008. 